\title{
Quality of life following surgery for congenital glaucoma: findings of the LVPEI congenital glaucoma registry
}

\author{
Vijaya K. Gothwal ${ }^{1} \cdot$ Bharani Seelam $^{1} \cdot$ Anil K. Mandal ${ }^{2}$
}

Received: 6 January 2018 / Revised: 10 September 2018 / Accepted: 15 October 2018 / Published online: 5 December 2018

(c) The Royal College of Ophthalmologists 2018

\begin{abstract}
Purpose To evaluate the health-related quality of life (HRQoL) of children operated for primary congenital glaucoma (PCG) using the Kidscreen-27 questionnaire.

Methods A total of 121 children (mean age, 11.8 years; SD, 2.8 years; 51\% males) with unilateral or bilateral PCG who underwent glaucoma surgery in infancy (mean duration since surgery, 10.2 years; [SD] 4.2 years) by a single surgeon at a tertiary eye care centre were administered the Kidscreen-27 questionnaire (self-or interviewer administered in a face-to-face interview) during their routine follow-up visit. We investigated the measurement properties of Kidscreen-27 questionnaire using Rasch analysis and generated interval-level estimates of HRQoL. The main outcome measure was HRQoL (i.e., Rasch-derived Kidscreen score). Multivariable linear regression analyses assessed the influence of socio-demographic and clinical variables on the HRQoL of children with PCG.

Results Majority (83\%) of the children had bilateral PCG and congenital type of the disease (79\%). Mean presenting acuity (logMAR) in the better eye was 0.32 (SD, 0.36; median, 0.18). Rasch analysis of Kidscreen-27 questionnaire indicated need for minor modifications following which a psychometrically robust unidimensional 23-item Kidscreen questionnaire emerged. In multivariable model, age of the child was independently associated with a $32 \%(\beta=-0.24[95 \% \mathrm{CI},-0.11$, $-0.36])$ increase in the HRQoL score.

Conclusions Despite undergoing successful glaucoma surgery, children with PCG reported reduced HRQoL. Younger children with PCG are more likely to experience this lowered HRQoL and ophthalmologists will need to be alert to this. Efforts must be made to improve the HRQoL of children with PCG.
\end{abstract}

\section{Introduction}

The aim of treatment for primary congenital glaucoma (PCG) is to reduce the intraocular pressure (IOP) and associated symptoms, enhance visual functioning, and improve well-being for the child and his or her immediate family members. Moreover, the measurement of treatment response is objective that includes measuring a reduction in IOP, and there have been several published reports including from our group regarding the visual and surgical

Anil K. Mandal

mandal@lvpei.org

1 Brien Holden Eye Research Centre-Patient-Reported Outcomes Unit, Hyderabad, India

2 Jasti V Ramanamma Children's Eye Care Centre, L V Prasad Eye Institute, Hyderabad, India outcomes of children operated for PCG [1-4]. However, the disease and its treatment may have an impact not only on visual functioning, but also on the overall well-being of the person, the 'quality of life (QoL)' [5]. QoL is a multidimensional construct comprising physical, psychological, social, and functional domains [6]. QoL as applied to medicine is more specifically known as 'health-related quality of life (HRQoL)' or 'subjective health status.' It measures the effect of illness, disease, and its treatment on the patient's welfare by going beyond the physician dominated indicators of the patient's progress [7, 8].

With recent shift towards assessment of health status and outcomes, the importance of HRQoL in measuring the health of individuals and populations is increasingly recognized as well as advocated $[9,10]$. Good quality of life (QoL) is a key outcome for the individual child, and is what parents want for their children. However little attention has been paid to the effect of PCG on the child's health and part of the reason for this may be related to issues with 
reliability of children's self-reports given their young age. Nonetheless, it has been demonstrated that children are able to self-report even at a young age [11-15]. Accurate measurement of QoL in children and adolescents is particularly important in the field of pediatric glaucoma so as to inform much of the utility of interventions. If interventions are not improving the well-being and QoL of children with PCG, the value of these interventions would be questionable. Hence, it is important to establish accurate measurement of QoL among those with PCG and to establish the psychometrics of these QoL measurements. Nonetheless, to study HRQL in PCG patients, first a decision has to be made about what measure to use. Currently, many HRQoL measures (generic and disease-specific [16]) for children are available and this affords an opportunity to measure the HRQoL of children with PCG comprehensively [17, 18]. Generic HRQoL measures have the advantage of facilitating the rating of a person and comparing it across conditions [19]. The Kidscreen-52 questionnaire is one such generic measure which is the first instrument for children and adolescents (aged 8-18 years) that was developed simultaneously in several different countries and tested in a large representative sample of children and adolescents, thereby helping to provide a broad perspective on the understanding and interpretation of HRQoL across different countries [20, 21]. Its measurement properties have been tested extensively, mostly using classical approaches and (to a limited extent) using modern item response theory [21]. Recently, shorter versions consisting of 27 and 10-items have been proposed [22, 23].

We conducted the present study with two aims: First, use the Kidscreen-27 questionnaire to investigate the HRQoL of children operated for PCG aged 8-18 years. Second, to identify socio-demographic variables associated with HRQoL of children with PCG. Given that the Kidscreen-27 questionnaire has not been validated in the PCG population and that it is important to have a psychometrically robust measure in order to derive valid conclusions, we investigated the psychometric properties of the Kidscreen-27 questionnaire.

\section{Methods}

\section{Participants}

We included consecutive patients aged 8-11 years (children) and 12-18 years (adolescents) with PCG (hereafter, both children and adolescents are referred to as 'children') who were operated for PCG by a single glaucoma specialist (AKM) between January 1997 and June 2012 at the LV Prasad Eye Institute, Hyderabad, India. Recruitment took place from January 2013 to March 2016. Other eligibility criteria included an ability to read/speak English or one of the local languages (Hindi or Telugu). Children with additional disabilities (such as hearing, cognitive impairment) were excluded. Primary congenital glaucoma was diagnosed according to generally accepted clinical signs, and primary combined trabeculotomy-trabeculectomy (CTT) was performed; simultaneous surgery was performed in both eyes in cases of bilateral PCG [1, 3, 24, 25]. Children with PCG were invited to participate in the study by the treating glaucoma specialist during their routine follow-up clinic visit. Each child underwent complete ophthalmic examination. The administration of the Kidscreen-27 questionnaire (see details of questionnaire later) took place in a quiet space away from the clinic and the researcher was available for any clarifications. Children were encouraged to complete the questionnaire by themselves but a single trained interviewer administered it in a face-to-face interview to those who couldn't self-administer (e.g. children couldn't read the font [Arial 16] on the questionnaire due to vision impairment or had pharmacological mydriasis). The role of the trained interviewer was to readout aloud the items on questionnaire and ask the child to choose from the options provided. Data collected from the medical records included: age of the child at surgery, date of surgery, laterality, and type of PCG, whether both or one eye was operated and clinical data, such as visual acuity and IOP.

The study was approved by the Ethics Committee for Human Research at the L V Prasad Eye Institute (LVPEI), Hyderabad, India, and followed the tenets of the Declaration of Helsinki.

\section{Kidscreen-27 questionnaire}

The Kidscreen-27 questionnaire is derived from the parent version-Kidscreen-52 that was developed using focusgroup work with children across 13 European countries [21, 26, 27]. In the present study, we chose to use the Kidscreen27 (child and adolescent self-report version) rather than the KIDSCREEN-52 so as to reduce the test burden for study participants. Table 1 contains the list of 27 items divided across five dimensions: physical well-being (5 items), psychological well-being (7 items), autonomy \& parent relations (7 items), social support \& peers (4 items), and school environment (4 items). The items utilize a 5-point Likerttype scale with three different sets of responses: (i) poor, fair, good, very good, excellent; (ii) not at all, slightly, moderately, very, extremely; (iii) never, seldom, quite often, very often, always. The response polarity was reversed according to standard procedures for four items to render them consistent with other items in the questionnaire whereby a higher score meant worse performance [28]. Higher (i.e., positive) Kidscreen-27 scores indicate worse 
Table 1 Item content of the Kidscreen-27 questionnaire

\begin{tabular}{ll}
\hline SL No. & Description \\
\hline $1^{*}$ & How is in general your health \\
$2^{\S}$ & Have you felt fit and well \\
$3^{*}$ & Have you been physically active \\
$4^{*}$ & Have you been able to run \\
$5^{*}$ & Have you felt full of energy \\
$6^{\dagger}$ & Have you enjoyed your life \\
$7^{\dagger}$ & Have you been in a good mood \\
$8^{\dagger}$ & Have you had fun \\
$9^{\dagger}$ & Have you felt sad \\
$10^{\dagger}$ & Have you felt so bad that you didn't want to do anything \\
$11^{\dagger}$ & Have you felt lonely \\
$12^{\dagger}$ & Have you been happy with the way you are \\
$13^{\Psi}$ & Have you had time for yourself \\
$14^{\Psi}$ & Have you had free time \\
$15^{\Psi}$ & Have your parents had enough time for you \\
$16^{\Psi \S}$ & Have your parents treated you fairly \\
$17^{\Psi}$ & Have you been able to talk to your parents \\
$18^{\Psi \S}$ & Have you had enough money to do the same things as \\
$19^{\Psi \S}$ & your friends \\
$20^{\zeta}$ & Have you had enough money for your expenses \\
$21^{\zeta}$ & Have you spent time with your friends \\
$22^{\zeta}$ & Have you and your friends helped each other \\
$23^{\zeta}$ & Have you been able to reply on your friends \\
$24^{\varphi}$ & Have you been happy at school \\
$25^{\varphi}$ & Have you got on well at school \\
$26^{\varphi}$ & Have you been able to pay attention \\
$27^{\varphi}$ & Have you got along with your teachers \\
\hline
\end{tabular}

*Physical well-being; ${ }^{\top}$ Psychological well-being; ${ }^{\Psi}$ Autonomy and parent relations; ${ }^{\Psi}$ Social support and peers; ${ }^{\zeta}$ School environment; ${ }^{\S}$ Items deleted (due to misfit) from Kidscreen-27 questionnaire using Rasch analysis in present study (see text for details)

HRQoL. The recall period is one week and responses measure how the respondent experienced each item during the previous week. Using standard guidelines for translation of questionnaires, we developed local language versions of the questionnaire for use in the present study [29]. The aim of this process was conceptual equivalence rather than a literal translation of the instrument for the two local languages (Hindi and Telugu). Briefly, the translation process consisted of two forward translations from target (English) to the source language (local language) by independent translators (fluent in English and the local language) which were later reconciled for any differences. The single reconciled version was then back translated into English by another translator independently (this person did not have access to the original English version and was not involved in the forward translation). Finally, cognitive debriefing was conducted on a representative sample of 6 children with PCG (3 each for Telugu and Hindi) and at this stage, every difficulty in understanding the questions, doubts, and suggestions of the participants were recorded. Few issues were raised by the participants on three items so changes were made to improve clarity: 'enough money for your expenses' was replaced with 'enough pocket money,' the words 'rely' and 'seldom' were replaced with 'believe,' and 'rarely', respectively, and 'got along well with your teachers' was replaced with 'satisfied with your teachers'. The revised instrument was administered to another group of 4 children with PCG who fully understood the contents of the instrument. The two final linguistically validated local language versions (Telugu and Hindi) along with the original version of the Kidscreen-27 were available for administration of the participants in the study.

\section{Assessment of measurement properties of Kidscreen-27 questionnaire}

We performed Rasch analysis on the Kidscreen-27 data using the Andrich rating-scale model [30] with Winsteps software (version 3.74) [31]. Rasch analysis is a form of item response theory that mathematically describes the interaction between the participants and items in a questionnaire and applies a probabilistic model that the pattern of participants' responses should satisfy. Details of Rasch analysis have been described in earlier publications by us [32-35]. Briefly, Rasch analysis converts ordinal ratings of questionnaire into estimates of interval measures data (expressed in log of the odds units, or logits). Rasch analysis also provides greater insight into the measurement properties of a questionnaire compared with CTT [36, 37]. Specifically, Rasch analysis permits investigation of the functioning of rating scale categories; the validity (i.e. does the questionnaire measure what it purports to measure) of a measure by evaluating the fit of individual items to the underlying construct (i.e. how well an individual item is in tandem with the whole group) and determining whether the items measure a unidimensional construct which is required to justify the summation of scores [38]; differential item functioning (DIF) which occurs when the likelihood of answering an item correctly by individuals with the same trait level varies according to their group allocation. We selected the DIF variables a priori in the present study for age (8-11 versus $12-18$ years), gender (male versus female) and laterality of PCG (unilateral versus bilateral), and was considered to be notable if $>1.0$ logits $[39,40]$.

\section{Statistical analysis}

We utilized the total Kidscreen questionnaire score linearly estimated using Rasch analysis in univariate and 
multivariate regression analysis, and used $t$-based $95 \%$ confidence intervals (CI) for the regression coefficients. A multivariate regression model was built using HRQoL score (i.e, Kidscreen score [interval level] in logits) as the dependent variable and age (in years), gender, laterality of PCG (unilateral/bilateral), duration since surgery (in years), type of PCG (congenital/infantile versus juvenile), presenting $\log$ MAR visual acuity (better-seeing eye) as independent variables. A forward stepwise strategy was applied to select significant independent variables with $p<0.05$ as the inclusion criterion. The purpose of these analyses was to examine the relationships between self-reported HRQoL (i.e., Kidscreen questionnaire scores) and socio-demographic variables. We estimated the difference in HRQoL between sub-groups and calculated effect size (ES) using the Cohen's $d$ statistic, which was categorized as small $(0.20)$, moderate $(0.50)$, or large $(0.80)$ [41]. All statistical tests were performed using IBM SPSS Statistics 19.0 software (IBM SPSS, Armonk, NY, USA). A $p$-value less than 0.05 was considered to be statistically significant.

\section{Results}

\section{Participant demographics}

Of the 912 children operated for PCG over the 15-year period, we included 121 children (222 eyes) in the present study. The majority had bilateral PCG (83\%) with congenital onset (79\%). The median age of the children at first glaucoma surgery was 5.5 months (interquartile range, 1.97-17.77 months). On initial presentation to the LVPEI, the majority of the children had PCG-related corneal haze ( $81 \%$ eyes), and $19 \%$ eyes had clear corneas (albeit with raised IOP). However, of those who had PCG-related corneal haze, the majority of the children (96\%) had clear cornea at the time of inclusion in the study. The mean (SD) preoperative IOP in the right and left eye was 28.1 (7.13) $\mathrm{mmHg}$ and 27.8 (6.77) $\mathrm{mmHg}$, respectively. At inclusion in the present study, the mean IOP in the right and left eye was $17.8(8.23) \mathrm{mmHg}$ and $17.2(7.48) \mathrm{mmHg}$, and there was a significant reduction of the IOP over the followup period $(P<0.0001$ for both right and left eyes). A little over one-half of the eyes (57\%) with PCG did not need any anti-glaucoma medication for control of IOP, 92 eyes $(41 \%)$ required 1 medication, and 3 eyes (1\%) underwent repeat trabeculectomy since their first glaucoma surgery. Approximately one-half of the children could selfadminister the questionnaire and a trained interviewer administered it to the remaining children $(54,45 \%)$. The average time for administration of the questionnaire was 30 min. The mean (SD) age of the children at inclusion
Table 2 Socio-demographic characteristics $(n=121)$

\begin{tabular}{lc}
\hline Characteristic & Result \\
\hline Mean age (SD), yrs & $11.8(2.8)$ \\
Male gender, $n(\%)$ & $62(51)$ \\
Mean duration since surgery (SD), yrs & $10.2(4.2)$ \\
Type of PCG, $n(\%)$ & \\
Congenital (birth to 1 month) & $95(79)$ \\
Infantile (>1 month to 3 years) & $10(8)$ \\
Juvenile (>3 years) & $16(13)$ \\
Laterality, $n(\%)$ & $20(17)$ \\
Unilateral & $101(83)$ \\
Bilateral & \\
Presenting visual acuity (logMAR better eye) & \\
Mean \pm SD & $0.32 \pm 0.36$ \\
Median & 0.18 \\
\hline
\end{tabular}

in the present study was 11.8 (2.8) years (Table 2) and their presenting acuity ranged from $20 / 20$ (logMAR 0.0 ) to light perception (logMAR 2.50) (median, $\log$ MAR 0.2 or Snellen equivalent 20/32).

\section{Psychometric evaluation of the Kidscreen-27 questionnaire}

Table 3 outlines the results of Rasch analysis of the Kidscreen-27 questionnaire. All the three types of rating scales displayed disordered thresholds, but merging adjacent response categories subsequently resulted in ordered thresholds for all. Four items that demonstrated misfit (i.e., infit residual values of $>1.3$ ) were iteratively deleted to improve fit statistics. Of these four misfitting items, two items also demonstrated large DIF by age (1.61 and 1.73 logits, respectively). Both the items were rated easier by children aged $12-18$ years as compared to their younger counterparts. Following deletion of four misfitting items, all the remaining 23 items fit the Rasch model and the psychometric properties of the revised questionnaire were adequate. Two items (Q3- "have you been physically active" and "have you been able to run well") were reported to be the least difficult. By comparison, the item, Q17- "have you been able to talk to your parents when you wanted to" was reported as the most difficult. All the five subscales had suboptimal measurement precision so these were considered dysfunctional and were not analyzed further. Therefore, we used the 23 items of Rasch-revised Kidscreen questionnaire to derive interval-level scores and used these for all our further analyses. The mean (SD) Kidscreen score of our participants was 2.22 (1.39) logits, and a positive score indicates lower HRQoL in the present study. 
Table 3 Overall performance of the native and revised Child and Adolescent versions of the Kidscreen-27 questionnaire

\begin{tabular}{llll}
\hline Parameter & Rasch model & $\begin{array}{l}\text { Kidscreen-27 } \\
\text { (native version) }\end{array}$ & $\begin{array}{l}\text { Kidscreen-23 } \\
\text { (revised version) }\end{array}$ \\
\hline $\begin{array}{l}\text { Number of items } \\
\text { Number of misfitting items }\end{array}$ & - & 27 & 23 \\
& 0 & 4 & $\begin{array}{l}\text { (4 misfitting } \\
\text { items deleted) }\end{array}$ \\
Person separation & $\geq 2.0$ & 1.88 & 2.22 \\
Reliability & $\geq 0.80$ & 0.78 & 0.83 \\
$\begin{array}{l}\text { Mean item location } \\
\text { Mean person location }\end{array}$ & 0 & 0 & 0 \\
$\begin{array}{l}\text { Principal components analysis } \\
\text { (eigenvalue for first contrast) }\end{array}$ & 0 & 1.16 & 2.22 \\
$\begin{array}{l}\text { Differential item functioning } \\
\text { (no. of items; }>1.0 \text { logit) }\end{array}$ & $\leq 3.0$ & 3.1 & 2.7 \\
\hline
\end{tabular}

\section{Relationship between HRQoL and PCG}

In the multiple linear regression model, only child's age was found to be statistically significantly associated with HRQoL (Table 4). This association was significant regardless of whether age was considered as a continuous variable (Table 4) or as a categorical variable (unstandardized $\beta$ coefficient -1.44 [95\% CI, $-0.87,-2.0], p<0.0001)$. Each 1 year increase in child's age was associated with a 0.28 logit (i.e. 32\%) increase (improvement) in his/her HRQoL. In the model, age explained $23 \%$ of the variance in the HRQoL of the participants. Subgroup analysis revealed that participants aged $8-11$ years reported significantly worse HRQoL as compared to those aged $12-18$ years $(\mathrm{ES}=0.44$; moderate ES).

\section{Discussion}

Our results demonstrated PCG had a negative impact on the HRQoL of children afflicted by this condition. Perhaps, a complex combination of disease onset during infancy and living with a chronic disease, the need for regular life-long hospital visits (resulting in frequent absenteeism from school), the need for use of anti-glaucoma medications or repeat surgeries (in some recalcitrant cases), and the fact that all these factors serve as a constant reminder of their eye disease may have affected the HRQoL of the children with PCG.

Our finding of lower HRQoL among younger compared with older children with PCG is consistent with that reported in the Western population, albeit using different questionnaire [42]. Recently, Dahlmann-Noor et al. used the Pediatric Quality of Life Inventory (PedsQL) to assess HRQoL of children with childhood glaucoma in the UK, albeit using raw questionnaire scores [42]. Using these raw ordinal scores, the authors reported variability and overlap in the HRQoL scores between the older (13-16 years) and
Table 4 Multiple regression analysis of factors associated with healthrelated quality of life of children with primary congenital glaucoma

\begin{tabular}{|c|c|c|c|c|}
\hline \multirow[t]{2}{*}{ Variable } & \multirow{2}{*}{$\begin{array}{l}\text { Unstandardized } \\
\text { coefficient }(\beta)\end{array}$} & \multirow[t]{2}{*}{ Sig. } & \multicolumn{2}{|c|}{$95 \%$ CI for $\beta$} \\
\hline & & & $\begin{array}{l}\text { Lower } \\
\text { bound }\end{array}$ & $\begin{array}{l}\text { Upper } \\
\text { bound }\end{array}$ \\
\hline (Constant) & 6.38 & $<0.0001$ & 4.52 & 8.25 \\
\hline Age & -0.24 & $<0.0001$ & -0.36 & -0.11 \\
\hline Male gender & 0.05 & 0.85 & -0.51 & 0.62 \\
\hline $\begin{array}{l}\text { Duration since } \\
\text { surgery }\end{array}$ & -0.04 & 0.32 & -0.13 & 0.04 \\
\hline Unilateral & -0.38 & 0.35 & -1.19 & 0.43 \\
\hline $\begin{array}{l}\text { Better eye visual } \\
\text { acuity (logMAR) }\end{array}$ & 0.34 & 0.18 & -0.15 & 0.83 \\
\hline $\begin{array}{l}\text { Congenital/ } \\
\text { infantile type } \\
\text { of primary } \\
\text { congenital } \\
\text { glaucoma }\end{array}$ & -0.73 & 0.16 & -0.18 & 0.29 \\
\hline
\end{tabular}

CI confidence interval, Sig. significance

younger children (5-7 and 8-12 years) [42]. Whereas the overall results are comparable between the UK study and ours, there are methodological differences. First, different questionnaires have been used. We utilized the Kidscreen27 questionnaire (child and adolescent version), a generic HRQoL instrument that has had high involvement of children and families in its development, assesses well-being and the items do not pose a threat to self-esteem of the child (items in the PedsQL focus on functioning with negatively stated items and if a child has lot of difficulty doing something then the items in the PedsQL may threaten self-esteem) [43, 44]. Second, the type of questionnaire scores used. In the present study, we validated the Kidscreen-27 questionnaire using Rasch analysis. By showing that our PCG data fit the Rasch model, we have empirically demonstrated that the Kidscreen scores in our sample possess estimates of interval-level measurement 
properties. This transformation is important as it reduces measurement noise and improves measurement precision that legitimizes the use of parametric statistical tests in analysis [33, 45], for e.g. comparison of scores before and after an intervention. The authors of the UK study used Likert or summary scoring and this method erroneously assumes that assigning ordinal numbers to response categories represents fundamental measurement and that all items in the questionnaire are of equal difficulty. Modern psychometric methods such as Rasch analysis have questioned these hypotheses [33]. Third, the sample characteristics are different between the two studies. In our study, we included cases with PCG only; majority had congenital type of PCG (79\%). By comparison, participants with both primary and secondary childhood glaucoma were included in the UK study; in the primary glaucoma category, most of the patients had infantile type PCG (32\%). In addition, they included patients who were awaiting glaucoma surgery (within 1 month of inclusion in the study) in addition to those who had undergone surgery. However we included patients who had undergone glaucoma surgery at least 8-9 years prior to being included in our study. Nevertheless, by combining the results presented here with those of the UK study, it would be safe to conclude that that the burden of living with PCG poses a considerable challenge to young children and adolescents (regardless of the ethnicity). Furthermore, regardless of the geographical location, with the passage of time, children and their families accommodate to having glaucoma such that their perception of the negative impact of glaucoma on the overall HRQoL aspects, including the physical, emotional, and social functioning decreases over time. This phenomenon has been observed in other chronic illness groups and has been labelled as "response shift" [46, 47]. It has been suggested that response shift may result from a change in an individual's internal standards, personal values, or conceptualization of perceived HRQoL and may involve behavioral, cognitive, and affective mechanisms or processes necessary for a person to accommodate to a changed health state [48]. The inherent limitations of assessment of a subjective concept such as QoL still remain in that there is still no means to ascertain that the fields questioned and the responses given truly represent the different life styles of differing communities, and hence the actual difficulties faced and the 'true, practical' impact of the disease on their life styles. Alternatively, given the cross-sectional nature of the data, this finding could be a function of survivor bias (i.e. older children with PCG who have had glaucoma for a long time may be better off in some ways). Longitudinal analysis of HRQoL in PCG will allow us to distinguish between these possibilities.
As values and priorities change during the life course, affected domains are likely to vary in different age groups. For example, although family and relationships with parents are more important for younger children, with increasing age, however other domains such as 'social support and peers' and 'school' become of growing importance [43]. However we could not separately assess the differences in various domains of the Kidscreen-27 questionnaire across the two age groups given that all subscales were dysfunctional. This indicates that subscale validity should not be assumed and should be tested using similar stringent criterion as that of the questionnaire as a whole. Given that person separation is sample dependent, the finding of dysfunctional subscales will, therefore, only be applicable in similar populations. Therefore, the validity of the subscales of the Kidscreen-27 should be tested in other populations. Assuming this sample is typical of a PCG population in the developing world, the likelihood of finding adequately performing subscales would, however, be low.

Although the Kidscreen-27 questionnaire was validated using Rasch analysis in several populations and has been shown to be reliable and valid for assessing HRQoL in different conditions, it has not been validated in PCG patients as yet [49]. Our study bridges this gap in the literature regarding the use of Kidscreen-27 as a HRQoL instrument in PCG patients. To our knowledge, this is the first time that Kidscreen-27 questionnaire has been subjected to Rasch analysis in an Indian PCG sample, and has resulted in a valid unidimensional measure of HRQoL in these patients. Of importance this finding highlights the need to re-validate questionnaires when used in a different population as has been performed for the Kidscreen-27 questionnaire in Colombian [50], Chinese [51], Persian populations [52]. Moreover, QoL is a subjective and abstract concept that deals with relationship between environment, and physiopsychological aspects of the individual, level of independence, social relationships, and personal beliefs. Therefore, while interpreting the QoL results across studies, attention should be paid to the differences in societal norms as well as cultural issues between different communities and ethnicities in different countries, and also among different locales within the same country. More importantly, as noted earlier, the inherent limitations associated with use of QoL questionnaires for the assessment of activities, abstracts, and thoughts across communities should also be borne in mind while interpreting the results of the present study.

A well-functioning questionnaire in a given population need not automatically imply optimal functioning in another population. For example, the Impact of Vision Impairment questionnaire for Children (IVI_C) questionnaire (developed in Australia) had to be modified 
when used in other populations, such as 17 item IVI-C in an Indian visually impaired pediatric population [15]. Overall, results from the present study demonstrate the Kidscreen questionnaire to be a reliable and valid tool to assess the impact of PCG on the HRQoL in our population, albeit with some minor changes: reduction in number of categories from 5 to 3 and deletion of four misfitting items. The need for reduction in number of categories is not unique and has been shown for the Persian version of the Kidscreen-27 questionnaire [52] and when used among childhood cancer survivors [53]. We believe that the three items misfit in our sample because they were measuring some other trait (two items tapping financial resources and one tapping parent relations) and therefore added noise (inaccuracy) in the measurement scale [54]. Item misfits have been reported for Kidscreen-27 questionnaire in populations with chronic conditions as well as in healthy samples. For example, Jervaues et al. used it among childhood cancer survivors and found five misfitting items and of these one item ('Have your parents treated you fairly'?) is common to our sample of PCG patients [53]. Recently, Shannon et al. used confirmatory factory analysis to test the psychometric properties of the Kidscreen-27 questionnaire in Irish children and demonstrated the need to split the 'autonomy and parent relations' (7 items) factor into an autonomy and parent relations factor [5 items] and financial resources factor [2 items] [55]. Similar observations were reported by $\mathrm{Ng}$ et al. using Chinese version of the questionnaire [51]. The Rasch-revised 23-item Kidscreen questionnaire in the present study conforms to the key fit indices for the Rasch model: unidimensionality, item fit, ordered thresholds, and lack of notable DIF.

Strengths of our study include the well-characterized large clinical sample of PCG representing different subtypes of the disease treated by a single glaucoma specialist over a 15-year period at a tertiary eye care centre and the use of Rasch analysis to validate the Kidscreen questionnaire in an Indian PCG sample so as to produce estimated linear interval of overall measure of HRQoL. When data satisfy Rasch model requirements, the approach transforms ordinal manifest data to latent interval-level data, which justifies the use of parametric statistics in the calculation of change scores, such as comparison of pre- and post-interventions. There were few limitations in this study. First, the cross-sectional design of the study does not allow for the inference of causal relationships. Second, the lack of a randomized control group, which means that the changes observed from pre- to post-surgery cannot be directly attributed to surgery. Third, although we made every attempt to approach consecutive patients as no potential participant refused participation, we may have inadvertently approached more willing patients and who may have had adequate resources to access eye care services (such as transportation, financial resources) and permission from their school to attend to their appointments. So sampling bias may have occurred, but recruitment according to preset demographic and/or clinical parameters may have resulted in a more representative cohort of patients. Finally, testretest reliability was not evaluated.

In conclusion, it is likely that children operated for PCG may have substantially compromised HRQoL despite surgical control of the disease. This is especially true for younger than for older children. This information can be supplemented to traditional clinical measures in the decision-making process when planning for any interventions for these patients. The Rasch-revised 23-item Kidscreen questionnaire is a psychometrically robust tool that is simple, brief, easily understood by children that can be used as a HRQoL outcome measure in the management of patients with PCG. In this way, the voices of the children will be heard in matters pertaining to their health and wellbeing given the perspective that 'some treatment effects are known only to the patient'. The use of HRQoL instruments such as the Kidscreen questionnaire brings the focus back on the patient rather than on disease and will be able to offer a good understanding of HRQoL from the PCG patient's perspective.

\section{Summary}

\section{What was known before}

- Children with primary congenital glaucoma undergo glaucoma surgery at a very early age necessitating lifelong follow-up. The aim of surgery for congenital glaucoma is to reduce the IOP, to decrease symptoms and to enhance visual functioning. However, congenital glaucoma and its treatment may have an impact not only on visual functioning, but also on the quality of life. However measurement of successful treatment response is often objective that includes measuring a reduction in IOP. Good quality of life is a key outcome for the individual child, and is what parents want for their children. Given the emphasis on controlling the IOP in congenital glaucoma, little attention has been paid to the impact of the disease on other aspects of a child's health. Several challenges exist when considering assessments of quality of life in children and the frequently cited barrier relates to the reliability of self-reports given their young age. Assessment of quality of life in children is particularly important in the field of pediatric glaucoma. This informs much of the utility of interventions. If interventions are not improving the quality of life of children with congenital glaucoma, the value of these interventions would be questionable. 


\section{What this study adds}

- After an average duration of 10 years, children operated for congenital glaucoma demonstrated controlled IOPs and over one-half recorded normal visual acuity (in better-seeing eye)suggesting good functional outcome, but they reported reduced health-related quality of life. Younger children (aged 8-11 years) with congenital glaucoma were more likely to report lowered healthrelated quality of life as compared with older children (aged 12-18 years). Kidscreen-27 questionnaire (Raschrevised child and adolescent version) demonstrated psychometrically robust properties in children operated for congenital glaucoma and could serve as a useful patient-reported outcome measure of health-related quality of life in this population. Children as young as 8 years can reliably report their quality of life. Ophthalmologists need to be aware of the adverse impact of congenital glaucoma on the child despite successful glaucoma surgery. Efforts must be made to periodically evaluate and improve the quality of life of children with congenital glaucoma.

Funding This study was supported in part by Hyderabad Eye Research Foundation, Hyderabad, India.

\section{Compliance with ethical standards}

Conflict of interest The authors declare that they have no conflict of interest.

\section{References}

1. Mandal AK, Gothwal VK, Bagga H, Nutheti R, Mansoori T. Outcome of surgery on infants younger than 1 month with congenital glaucoma. Ophthalmology. 2003;110:1909-15.

2. Mandal AK, Naduvilath TJ, Jayagandan A. Surgical results of combined trabeculotomy-trabeculectomy for developmental glaucoma. Ophthalmology. 1998;105:974-82.

3. Mandal AK, Gothwal VK, Nutheti R. Surgical outcome of primary developmental glaucoma: a single surgeon's long-term experience from a tertiary eye care centre in India. Eye. 2007;21:764-74.

4. Mendicino ME, Lynch MG, Drack A, Beck AD, Harbin T, Pollard Z, et al. Long-term surgical and visual outcomes in primary congenital glaucoma: 360 degrees trabeculotomy versus goniotomy. J AAPOS. 2000;4:205-10.

5. O'Boyle CA. Assessment of quality of life in surgery. Br J Surg. 1992;79:395-8

6. Neugebauer E, Troidl H, Wood-Dauphinee S, Eypasch E, Bullinger M. Quality of life assessment: results of the Meran Consensus Development Conference. Theor Surg. 1991;6: 123-37.

7. Cohen C. On the quality of life: some philosophical reflections. Circulation. 1982;66:29-33.
8. Gotay CC, Korn EL, McCabe MS, Moore TD, Cheson BD. Quality-of-life assessment in cancer treatment protocols: research issues in protocol development. J Natl Cancer Inst. 1992;84: 575-9.

9. Straatsma BR. Clinical trials in the past 25 years and clinical research in the next 25 years. Am J Ophthalmol. 1999;127:72-74.

10. Guyatt GH, Feeny DH, Patrick DL. Measuring health-related quality of life. Ann Intern Med. 1993;118:622-9.

11. Feeny D, Furlong W, Boyle M, Torrance GW. Multi-attribute health status classification systems. Health Utilities Index. Pharmacoeconomics. 1995;7:490-502.

12. Juniper EF, Guyatt GH, Feeny DH, Griffith LE, Ferrie PJ. Minimum skills required by children to complete health-related quality of life instruments for asthma: comparison of measurement properties. Eur Respir J. 1997;10:2285-94.

13. Cremeens J, Eiser C, Blades M. Factors influencing agreement between child self-report and parent proxy-reports on the Pediatric Quality of Life Inventory 4.0 (PedsQL) generic core scales. Health Qual Life Outcomes. 2006;4:58.

14. Gothwal VK, Lovie-Kitchin JE, Nutheti R. The development of the LV Prasad-Functional Vision Questionnaire: a measure of functional vision performance of visually impaired children. Invest Ophthalmol Vis Sci. 2003;44:4131-9.

15. Gothwal VK, Sumalini R, Irfan SM, Giridhar A. Rasch analysis of Impact of Vision Impairment for Children questionnaire. Optom Vision Sci. 2013;90:820-7.

16. Solans M, Pane S, Estrada MD, Serra-Sutton V, Berra S, Herdman $\mathrm{M}$, et al. Health-related quality of life measurement in children and adolescents: a systematic review of generic and diseasespecific instruments. Value Health. 2008;11:742-64.

17. Eiser C, Morse R. A review of measures of quality of life for children with chronic illness. Arch Dis Child. 2001;84:205-11.

18. Harding L. Children's quality of life assessment: a review of generic and health-related quality of life measures completedby children and adolescents. Clin Psychol Psychother. 2001;8:79-96.

19. Varni JW, Seid M, Rode CA. The PedsQL: measurement model for the pediatric quality of life inventory. Med care. 1999;37: 126-39.

20. Ravens-Sieberer U, Erhart M, Power M, Auquier P, Cloetta B, Hagquist $C$, et al. Item-response theory analyses of child and adolescent self-report quality of life data: the European cross-cultural research instrument KIDSCREEN. Qual Life Res. 2003; 12:722.

21. Ravens-Sieberer U, Gosch A, Rajmil L, Erhart M, Bruil J, Duer W, et al. KIDSCREEN-52 quality-of-life measure for children and adolescents. Expert Rev Pharm Outcomes Res. 2005;5:353-64.

22. Robitail S, Ravens-Sieberer U, Simeoni MC, Rajmil L, Bruil J, Power M, et al. Testing the structural and cross-cultural validity of the KIDSCREEN-27 quality of life questionnaire. Qual Life Res. 2007;16:1335-45.

23. Ravens-Sieberer U, Erhart M, Rajmil L, Herdman M, Auquier P, Bruil $\mathbf{J}$, et al. Reliability, construct and criterion validity of the KIDSCREEN-10 score: a short measure for children and adolescents' well-being and health-related quality of life. Qual Life Res. 2010;19:1487-1500.

24. Mandal AK, Bhatia PG, Bhaskar A, Nutheti R. Long-term surgical and visual outcomes in Indian children with developmental glaucoma operated on within 6 months of birth. Ophthalmology. 2004;111:283-90.

25. Mandal AK, Netland P. The Pediatric Glaucomas. Philadelphia: Elsevier; 2006.

26. Ravens-Sieberer U, Gosch A, Abel T, Auquier P, Bellach BM, Bruil J, et al. Quality of life in children and adolescents: a European public health perspective. Sozial- und Prav. 2001;46: 294-302. 
27. Ravens-Sieberer U, Gosch A, Rajmil L, Erhart M, Bruil J, Power M, et al. The KIDSCREEN-52 quality of life measure for children and adolescents: psychometric results from a crosscultural survey in 13 European countries. Value Health. 2008;11: 645-58.

28. The KIDSCREEN Group Europe. The KIDSCREEN Questionnaires Quality of Life questionnaires for children and adolescents Handbook Lengerich, Germany: Pabt Science Publishers; 2006.

29. Guillemin F, Bombardier C, Beaton D. Cross-cultural adaptation of health-related quality of life measures: literature review and proposed guidelines. J Clin Epidemiol. 1993;46:1417-32.

30. Andrich DA. A rating scale formaultion for ordered response categories. Psychometrika. 1978;43:361-73.

31. Linacre JM. A User's Guide to Winsteps/Ministeps Rasch Model Computer Programs. Chicago, IL: MESA Press; 2014.

32. Massof RW. The measurement of vision disability. Optom Vision Sci. 2002;79:516-52.

33. Pesudovs K, Burr JM, Harley C, Elliott DB. The development, assessment, and selection of questionnaires. Optom Vision Sci. 2007;84:663-74.

34. Gothwal VK, Wright TA, Lamoureux EL, Pesudovs K. Activities of Daily Vision Scale: what do the subscales measure? Invest Ophthalmol Vis Sci. 2010;51:694-700.

35. Gothwal VK, Bharani S, Mandal AK. Quality of life of caregivers of children with congenital glaucoma: development and validation of a novel questionnaire (CarCGQoL). Invest Ophthalmol Vis Sci. 2015;56:770-7.

36. Bond TG, Fox CM. Appplying the Rasch Model: Fundamental Measurement in the Human Sciences. London: Lawrence Erlbaum Associates; 2001.

37. Wright BD. Masters GN Rating scale analysis. Chicago: MESA Press; 1982.

38. Emberston SE, Reise SP. Item Response Theory for Psychologists. Marwah, NJ: Erlbaum Associates; 2000.

39. Wright BD. Best Test Design: Rasch Measurement. Chicago: MESA Press; 1979.

40. Wright BD, Douglas GA. Best test design and self-tailored testing. Chicago, IL: Statistical laboratory, Department of Education, University of Chicago; 1975.

41. Cohen JW. Statistical power analysis for the behavioural sciences. Hillsdale, NJ: Lawrence Earlbaum Associates; 1988.

42. Dahlmann-Noor A, Tailor V, Bunce C, Abou-Rayyah Y, Adams G, Brookes J, et al. Quality of life and functional vision in children with glaucoma. Ophthalmology. 2017;124: $1048-55$.
43. Gringeri A, von Mackensen S, Auerwald G, Bullinger M, PerezGarrido R, Kellermann E, et al. Haemo-QoL Study. Health status and health-related quality of life of childen with haemophilia from six West European countries. Haemopilia. 2004;10: 26-33.

44. Petersson C, Simeonsson RJ, Enskar K, Huus K. Comparing children's self-report instruments for health-related quality of life using the International Classification of Functioning, Disability and Health for Children and Youth (ICF-CY). Health Qual Life Outcomes. 2013;11:75.

45. Svensson E. Guidelines to statistical evaluation of data from rating scales and questionnaires. J Rehabil Med. 2001;33:47-48.

46. Saigal S, Tyson J. Measurement of quality of life of survivors of neonatal intensive care: critique and implications. Semin Perinatol. 2008;32:59-66.

47. Parsons SK, Barlow SE, Levy SL, Supran SE, Kaplan SH. Healthrelated quality of life in pediatric bone marrow transplant survivors: according to whom? Int J Cancer Suppl. 1999;12:46-51.

48. Sprangers MA, Schwartz CE. Integrating response shift into health-related quality of life research: a theoretical model. Soc Sci Med. 1999;48:1507-15.

49. Lamoureux EL, Pallant JF, Pesudovs K, Hassell JB, Keeffe JE. The Impact of Vision Impairment Questionnaire: an evaluation of its measurement properties using Rasch analysis. Invest Ophthalmol Vis Sci. 2006;47:4732-41.

50. Velez CM, Lugo-Agudelo LH, Hernandez-Herrera GN, GarciaGarcia HI. Colombian Rasch validation of KIDSCREEN-27 quality of life questionnaire. Health Qual Life Outcomes. 2016;14:67.

51. Ng JY, Burnett A, Ha AS, Sum KW. Psychometric properties of the Chinese (Cantonese) versions of the KIDSCREEN healthrelated quality of life questionnaire. Qual Life Res. 2015;24: 2415-21.

52. Jafari P, Safe M. Item and response-category functioning of the Persian version of the KIDSCREEN-27:Rasch partial credit model. Health Qual Life Outcomes. 2012;10:127.

53. Jervaeus A, Kottorp A, Wettergren L. Psychometric properties of KIDSCREEN-27 among childhood cancer survivors and age matched peers: a Rasch analysis. Health Qual Life Outcomes. 2013;11:96.

54. Smith RM, Suh KK. Rasch fit statistics as a test of the invariance of item parameter estimates. J Appl Meas. 2003;4:153-63.

55. Shannon S, Breslin G, Fitzpatrick B, Hanna D, Brennan D. Testing the psychometric properties of Kidscreen-27 with Irish children of low socio-economic status. Qual Life Res. 2017;26: 1081-9. 